\title{
Allergic Broncho Pulmonary Aspergillosis Complicated by Nocardiosis
}

\author{
Brijesh Sharma, ${ }^{1}$ Gopal Ghosh, ${ }^{1}$ Ulka Kamble, ${ }^{1}$ Karan Chaudhary, ${ }^{1}$ Ajay Chauhan, ${ }^{1}$ \\ B. M. S. Lamba, ${ }^{1}$ Anuradha Chowdhary, ${ }^{2}$ and B. B. Gupta ${ }^{1}$ \\ ${ }^{1}$ Department of Medicine, PGIMER \& Dr. Ram Manohar Lohia Hospital, New Delhi 110001, India \\ ${ }^{2}$ Department of Medical Mycology, V.P. Chest Institute, University of Delhi, Delhi 110007, India \\ Correspondence should be addressed to Brijesh Sharma, brijsushma@gmail.com
}

Received 19 September 2012; Accepted 12 December 2012

Academic Editors: D. Franzen, M. Ip, and S. A. McGrath-Morrow

Copyright (c) 2012 Brijesh Sharma et al. This is an open access article distributed under the Creative Commons Attribution License, which permits unrestricted use, distribution, and reproduction in any medium, provided the original work is properly cited.

We describe a 70-year-old male with a history of diabetes mellitus, hypertension, and asthma who presented with increasing breathlessness for 5 months. He was diagnosed to have allergic bronchopulmonary aspergillosis (ABPA) by serological and radiographic criteria. He was treated with steroids and itraconazole. After initial improvement, he developed fever with cough and mucopurulent sputum. X-ray chest revealed multiple cavities with air fluid level. Patient was treated with antibiotics without any response. Sputum was negative for acid fast bacilli (AFB). Sputum culture for bacteria and fungus did not reveal any significant growth; however a delayed growth of Nocardia was noted on fungal plates. Modified Ziehl Nelsen stain was positive for AFB. Patient was treated with cotrimoxazole. We discuss the serological and radiological criteria of ABPA, presentation and treatment of nocardia pulmonary infection and other possible causes of necrotizing pneumonia in immunocompromised settings.

\section{Case Discussion}

A 70-year-old male with a history of bronchial asthma for 25 years, type 2 diabetes, and hypertension for 10 years presented with a 5-month history of increasing breathlessness. $\mathrm{He}$ had seen multiple physicians over this period and had been treated with various antibiotics, ciprofloxacin, levofloxacin, and, most recently amoxicillin/clavulanic acid and inhaled and oral bronchodilators with little to no improvement. At the time of admission, he complained of increasing shortness of breath for last 5 days, fever with chills for 3 days, minimally productive cough, and generalized fatigue. Over the years he had been treated with inhaled formoterol and fluticasone for asthma. He denied chronic systemic corticosteroid use. His diabetes was not controlled, though he was taking oral hypoglycaemic agents (metformin and glimepiride). His hypertension was under control on amlodipine. He had no known drug allergies and denied any family history of lung disease or allergies. There was no history of exposure to animals, environmental irritants, or tuberculosis. He denied high risk sexual behaviour and tobacco, alcohol, or intravenous drug use.

On physical examination, he was febrile with temperature of $100.4^{\circ} \mathrm{F}$, pulse rate of $94 /$ minutes, blood pressure of $130 / 78 \mathrm{~mm}$ of $\mathrm{Hg}$, respiratory rate of 20 breaths per min with accessory muscle use, and oxygen saturation of $94 \%$ on room air. Respiratory system examination revealed bilateral inspiratory and expiratory wheeze and bibasilar crackles, and bronchial breathing in right infraclavicular region.

On investigation chest radiogram (Figure 1) revealed fibrotic opacities in bilateral lung fields mainly in mid and lower zones, prominent on right side with cavitary lesions in right mid and upper zones.

HRCT (high resolution computed tomography) of chest (Figures 2 and 3 ) revealed extensive emphysematous changes in both lung fields with bulla formation, central bronchiectatic changes in anterior, apical, and posterior segments of right upper lobe, apicoposterior segment of left upper lobe, lateral basal segment of right lower lobe, and apical segment of left lower lobe with peribronchial wall thickening. 


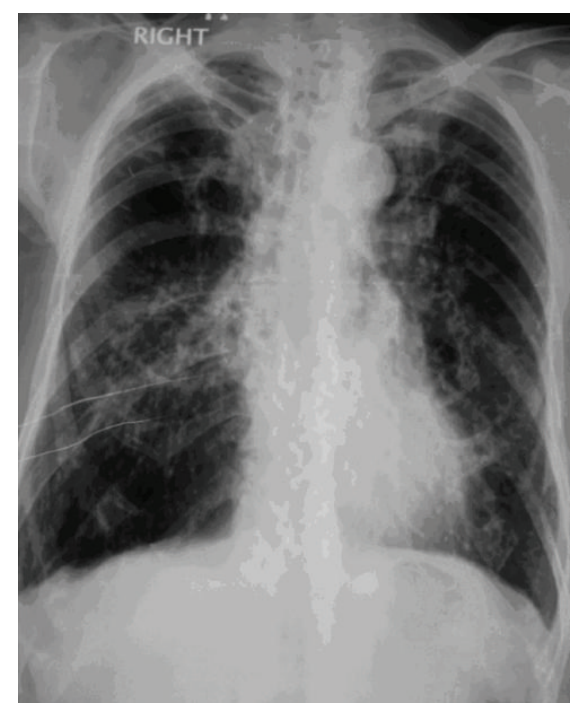

Figure 1

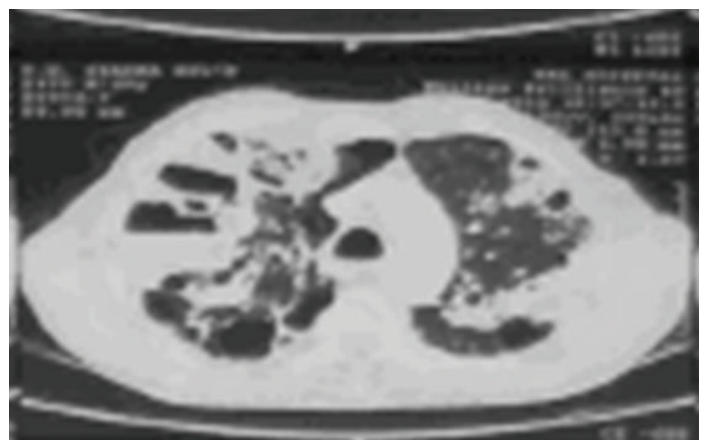

Figure 2

We proceeded for further investigations. Ziehl-neelsen staining of sputum was negative for acid fast bacilli on several occasions and sputum for gram staining was negative for any pathogen and sputum $\mathrm{KOH}$ preparation failed to demonstrate any fungal hyphae. Serum immunoglobulin $\mathrm{G}$ specific for Aspergillus fumigatus was raised $(192.68 \mathrm{iu} / \mathrm{mL})$. Total serum immunoglobulin E level was $6000 \mathrm{iu} / \mathrm{mL}$ (markedly elevated). Patient was labelled as a case of ABPA and started on steroid with itraconazole. He showed initial improvement. Breathlessness and daily activity improved. Patient was shifted to insulin for better glycemic control. He was discharged and was advised followup in outpatient clinic.

Three week after the treatment started, patient again presented to us with complaints of high grade fever with cough and mucopurulent expectoration and increased shortness of breath accompanied by pleuritic chest pain. On examination patient was restless with respiratory rate of 30 breaths/minute, blood pressure of $130 / 74 \mathrm{~mm} \mathrm{Hg}$, pulse rate of $96 /$ minute, and temperature of $102^{\circ} \mathrm{F}$. There was no pallor, icterus, cyanosis, clubbing, oedema, and lymphadenopathy. Oxygen saturation was $90 \%$ on room air. Respiratory examination revealed bilateral coarse crackles with expiratory wheezing with amphoric bronchial breath sounds in

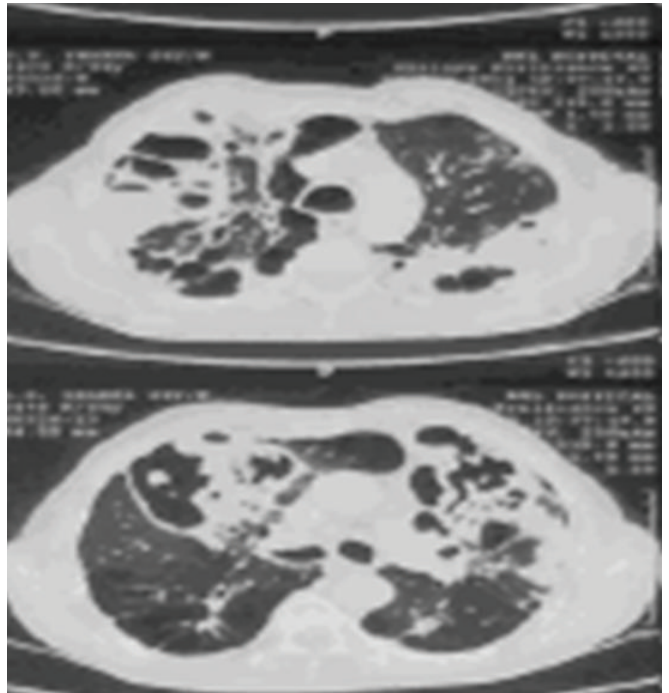

Figure 3

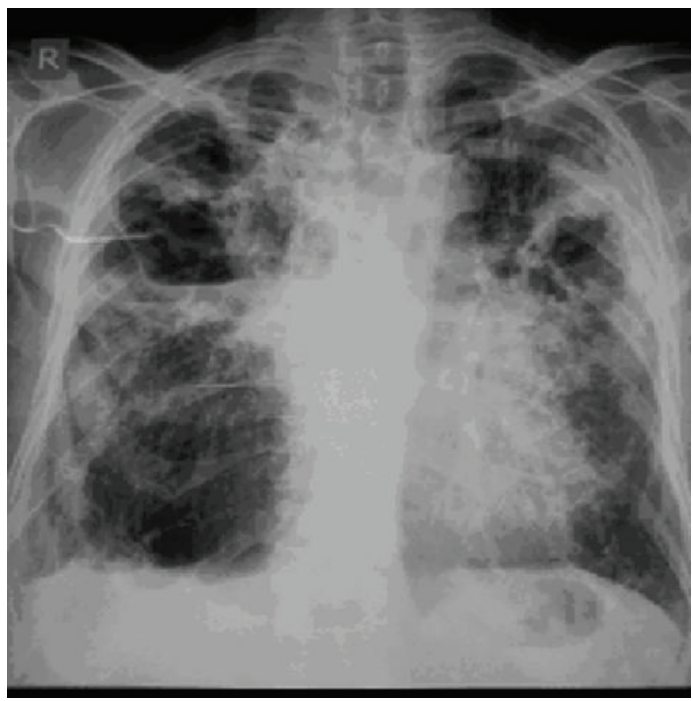

FIgURe 4

right infraclavicular and suprascapular areas. Steroid was withheld, itraconazole was continued, patient was stabilized with oxygen, and nebulised bronchodilators and antibiotics were added. Patient was again investigated. Investigations revealed hemoglobin of $11.7 \mathrm{gm} \%$, total leukocyte count of $17000 / \mathrm{mm}^{3}$, peripheral smear showed toxic granules, kidney and liver function test results were within normal limits, Xray chest (Figure 4) revealed multiple cavitary lesions with air fluid levels in right upper and mid zones and left mid zone with adjacent area of consolidation, fibrosis and volume loss. Inhomogeneous infiltrates were noted in left lower zone. Repeated Ziehl-neelsen stain of sputum were negative for AFB. Sputum for fungal culture showed insignificant fungal growth, repeated sputum for bacterial culture showed no growth. However delayed growth of Nocardia was noted on fungal plates. Sputum sample was sent for modified acid fast staining which showed the Nocardia asteroids (Figure 5) as 


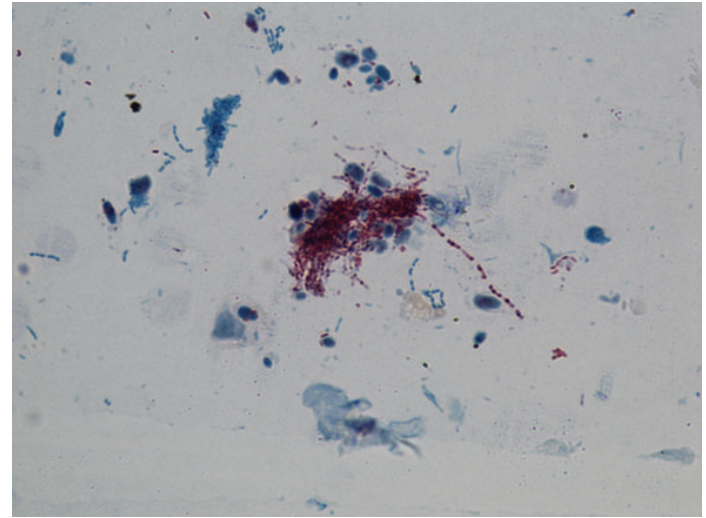

FIGURE 5

weakly acid fast positive filaments. Patient was started on tablet cotrimoxazole in dose of sulphamethoxazole $2400 \mathrm{mg}$ and trimethoprim $480 \mathrm{mg}$ per day in divided doses. Patient became symptomatically well within a week of starting cotrimoxazole and was discharged. Patient was completely asymptomatic after one month of therapy.

\section{Discussion}

The minimum essential criteria for diagnosis of ABPA in asthma are the presence of asthma, immediate cutaneous reactivity to Aspergillus species or Aspergillus fumigatus, an elevated total serum $\operatorname{IgE}(>417 \mathrm{kU} / \mathrm{l})$, and elevated serum IgG or IgE to Aspergillus fumigatus. A diagnosis of seropositive ABPA (ABPA-S) can be made with the above criteria [1]. Radiographic findings further categorize the disease. Presence of ABPA-S plus central bronchiectasis, that is, bronchiectasis in inner two-thirds of chest CT field, is necessary for a diagnosis of ABPA with central bronchiectasis (ABPA-CB). A recent article designated a third variation of this process as ABPA with central bronchiectasis and other radiologic features (ABPA-CB-ORF). These patients, in addition to satisfying criteria for ABPA-CB also had other radiologic features on CT such as pulmonary fibrosis, blebs, scarring, and fibrocavitary lesions. With progression from ABPA$\mathrm{S}$ to ABPA-CB-ORF, the disease becomes increasingly severe [2].

On initial presentation, our patient fulfilled the criteria of ABPA-CB-ORF and hence we initiated the treatment for the same. The patient came back after three weeks with worsening of symptoms. Patient was reevaluated as the X-ray chest showed multiple cavitary lesions with air fluid levels and evidence of sepsis (polymorph nuclear leucocytosis with toxic granules, fever, and tachypnoea). So our patient was investigated on the line of lung abscess with reactivation of tuberculosis and invasive aspergillosis as differential diagnosis. Repeated sputum for gram stain and bacterial culture were sterile, sputum smears for Ziehl-Neelsen staining were negative for acid fast organisms, and sputum for fungal culture showed insignificant growth. We reviewed the literature for cavitatory lesions in immunodeficient individuals, and found that pneumonia is typically caused by Staphylococcus aureus [3] or Pseudomonas aeruginosa [4], and Mycobacterium tuberculosis [5-7] is rarely reported with Rhodococcus equi [8-10] and Nocardia asteroids [11-17].

Staphylococcal pneumonia [3] is frequently severe and typically occurs in relatively debilitated patients. Predisposing factors are age > 65 years, alcoholism, chronic bronchopulmonary disease, immunodepression, renal failure, and diabetes [18]. Pseudomonal pneumonia typically occurs in the setting of cystic fibrosis and other immunocompromised settings but its exact association in ABPA setting is still unknown. Both of them are readily cultured on Blood Agar medium. Tuberculosis is the commonest opportunistic infection in HIV infected patients; other risk factors for tuberculosis are birth in a country where tuberculosis is endemic, diabetes, presence of head and neck malignancy, haematologic malignancy, corticosteroid use, and treatment with tumor necrosis factor inhibitor [5-7]. Sputum smear with ZN staining is positive and more so in cavitatory disease. Rhodococcus equi is a gram-positive coccobacillus commonly isolated from soil, common cause of pneumonia in young horses. Risk factors in human are advanced human immunodeficiency virus infection (CD4 T-lymphocyte count of 200 cells $/ \mathrm{mm}^{3}$ or less), hematologic malignancies, and use of chronic corticosteroids and other immunosuppressive agents [8-10]. No case has ever been reported in India.

Majority of patients with pulmonary nocardiosis have identifiable risk factors mainly corticosteroid therapy and other immunosuppressant therapy or some evidence of immunosuppression [12]. Nocardiosis is a life-threatening infection [17]. The association between nocardiosis and solid organ transplantation [14], HIV infection with CD4 cell count $<50 /$ cumm [15], male gender [16], steroid therapy, and immunosuppressant therapy [12] has been observed in few studies. COPD is common respiratory disease among patients with pulmonary nocardiosis [13]. COPD is commonly treated with corticosteroids. Patients have frequent infections. Bacteria colonizing the lower airways alter ciliary motility and cause epithelial damage, thereby facilitating the presence of Nocardia [11]. A similar phenomenon is found in patients with cystic fibrosis and bronchiectasias, who also present with epithelial changes [11]. Our patient was a longstanding asthmatic, who had developed COPD. His HRCT revealed extensive emphysematous changes in both lung with bullae formation, extensive bronchiectatic changes with peribronchial wall thickenings. He had developed ABPA-CB-ORF and had been treated with corticosteroids. He was also a diabetic, thus he had all the risk factors to develop pulmonary nocardiosis.

The clinical symptoms and CXR findings of pulmonary nocardiosis are nonspecific [19], similar to those caused by other bacteria $[20,21]$. The illness tends to have a protracted course and the diagnosis is often delayed [22]. To reduce the delay in diagnosis and treatment, testing for Nocardia spp. should be performed in patients with risk factors for pulmonary nocardiosis and pneumonia which have not responded to treatment. Microbiological studies of respiratory tract specimens obtained by noninvasive methods are effective [23]. However, if the patient cannot expectorate, 
respiratory tract specimens obtained by invasive methods have a reasonable yield [23, 24]. Microscopy and culture are not difficult [22]; however, because of the slow growth of the organisms, cultures should be kept for at least 30 days $[22,24]$. The microbiology laboratory should be informed that Nocardia spp. is suspected as the bacteria require specific media, a long culture period and modified Ziehl-nelsen staining for direct demonstration [24, 25]. In immunosuppressed patients, a positive sputum culture is more likely to indicate disease and not colonization, and the patients must be treated. Sulphonamides have been the mainstay of therapy of Nocardiosis. Trimethoprim sulphamethoxazole (TMP-SMX) combination has been usually used for treatment. There have been reports of poor treatment responses in patients of nocardiosis treated with TMP-SMX combination alone [26, 27]. Combination therapies with cotrimoxazole, amikacin, and cephalosporin or imipenem have been recommended as empirical therapy in serious, CNS, and disseminated cases [28]. Linzeolid offers an additional option. Therapy is given for a protracted period as relapses may occur [29]. There are few case reports of TMP-SMX resistance in Nocardia spp. from India [26] and most of the isolates even of Nocardia farcinica are susceptible to TMP-SMX [30]. Our patient had only pulmonary nocardiosis and showed a good response to treatment with TMP-SMX alone. He has been treated for 16 weeks and is doing well after finishing treatment.

\section{Conclusion}

All the patients with the risk factors like COPD, bronchiectasis, cystic fibrosis, and any other immunocompromised state, with nonresponsive pneumonia must be evaluated for rare infections like nocardia. Delay in diagnosis and treatment can be life threatening. Resistance is an emerging concern and combination therapy is an option in serious and disseminated disease.

\section{References}

[1] R. Kumar, "Mild, moderate, and severe forms of allergic bronchopulmonary aspergillosis: a clinical and serologic evaluation," Chest, vol. 124, no. 3, pp. 890-892, 2003.

[2] R. Agarwal, A. Khan, D. Gupta, A. N. Aggarwal, A. K. Saxena, and A. Chakrabarti, "An alternate method of classifying allergic bronchopulmonary aspergillosis based on high-attenuation mucus," PLoS ONE, vol. 5, no. 12, Article ID e15346, 2010.

[3] Y. Gillet, P. Vanhems, G. Lina et al., "Factors predicting mortality in necrotizing community-acquired pneumonia caused by Staphylococcus aureus containing panton-valentine leukocidin," Clinical Infectious Diseases, vol. 45, no. 3, pp. 315-321, 2007.

[4] M. A. Gharabaghi, S. M. Abdollahi, E. Safavi et al., "Community acquired Pseudomonas pneumonia in an immune competent host," BMJ Case Reports, vol. 26, article 2012, 2012.

[5] S. Patel, A. E. Parsyan, J. Gunn et al., "Risk of progression to active tuberculosis among foreign-born persons with latent tuberculosis," Chest, vol. 131, no. 6, pp. 1811-1816, 2007.

[6] S. J. Kim, Y. P. Hong, W. J. Lew et al., "Incidence of pulmonary tuberculosis among diabetics," Tubercle and Lung Disease, vol. 76, pp. 529-533, 1995.
[7] American Thoracic Society, "Targeted tuberculin testing and treatment of latent tuberculosis infection," MMWR-Recommendations and Reports, vol. 49, pp. 1-51, 2000.

[8] K. J. Gray, N. French, E. Lugada, C. Watera, and C. F. Gilks, "Rhodococcus equi and HIV-1 infection in Uganda," Journal of Infection, vol. 41, no. 3, pp. 227-231, 2000.

[9] E. A. Garthwaite, D. J. Border, C. H. Jones, and D. P. Worth, "Rhodococcus equi infection during treatment of a C-ANCA positive vasculitis: a case report," Rheumatology International, vol. 27, no. 3, pp. 285-287, 2007.

[10] O. Lortholary, J. L. Mainardi, B. La Scola, V. Gallais, P. Frenaux, and P. Casassus, "Consecutive bacillary angiomatosis and Rhodococcus equi bacterimia during acute leukemia: zoonoses may cause fever in neutropenic patients," Clinical Microbiology and Infection, vol. 6, no. 6, pp. 334-336, 2000.

[11] F. Ader, S. Nseir, R. Le Berre et al., "Invasive pulmonary aspergillosis in chronic obstructive pulmonary disease: an emerging fungal pathogen," Clinical Microbiology and Infection, vol. 11, no. 6, pp. 427-429, 2005.

[12] M. V. Minero, M. Marín, E. Cercenado, P. M. Rabadán, E. Bouza, and P. Muñoz, "Nocardiosis at the turn of the century," Medicine, vol. 88, no. 4, pp. 250-261, 2009.

[13] R. Martínez Tomás, R. Menéndez Villanueva, S. Reyes Calzada et al., "Pulmonary nocardiosis: risk factors and outcomes," Respirology, vol. 12, pp. 394-400, 2007.

[14] A. Y. Peleg, S. Husain, Z. A. Qureshi et al., "Risk factors, clinical characteristics, and outcome of Nocardia infection in organ transplant recipients: a matched case-control study," Clinical Infectious Diseases, vol. 44, no. 10, pp. 1307-1314, 2007.

[15] V. Pintado, E. Gómez-Mampaso, J. Cobo et al., "Nocardial infection in patients infected with the human immunodeficiency virus," Clinical Microbiology and Infection, vol. 9, no. 7, pp. 716-720, 2003.

[16] H. Al-Jahdali, S. Baharoon, S. Alothman, Z. Memish, and A. Waness, "Nocardiosis in a tertiary care hospital in Saudi Arabia," Journal of Global Infectious Diseases, vol. 3, no. 2, pp. 128-132, 2011.

[17] X. Yu, F. Han, J. Wu et al., "Nocardia infection in kidney transplant recipients: case report and analysis of 66 published cases," Transplant Infectious Disease, vol. 13, no. 4, pp. 385391, 2011.

[18] P. Germaud, S. Caillet, J. Caillon, and M. C. Allenet, "Community acquired Staphylococcus aureus pneumonia in non-HIV infected adults," Revue de Pneumologie Clinique, vol. 55, no. 2, pp. 83-87, 1999.

[19] J. H. Hwang, W. J. Koh, G. Y. Suh et al., "Pulmonary nocardiosis with multiple cavitary nodules in a HIV-negative immunocompromised patient," Internal Medicine, vol. 43, no. 9, pp. 852-854, 2004.

[20] B. Q. Wu, T. T. Zhang, J. X. Zhu, H. Liu, J. Huang, and W. X. Zhang, "Pulmonary nocardiosis in immunocompromised host: report of 2 cases and review of the literature," Zhonghua Jie He He Hu Xi Za Zhi, vol. 32, no. 8, pp. 593-597, 2009.

[21] J. Ambrosioni, D. Lew, and J. Garbino, "Nocardiosis: updated clinical review and experience at a tertiary center," Infection, vol. 38, no. 2, pp. 89-97, 2010.

[22] E. R. Lederman and N. F. Crum, "A case series and focused review of nocardiosis: clinical and microbiologic aspects," Medicine, vol. 83, no. 5, pp. 300-313, 2004.

[23] N. K. Patel, L. Snyder et al., "Pulmonary nocardiosis and empyema in a patient with metastatic neuroendocrine tumor," Southwest Jounrnal of Pulmonary and Critical Care, vol. 3, pp. 28-33, 2011. 
[24] G. S. Gaude, B. M. Hemashettar, A. S. Bagga, and R. Chatterji, "Clinical profile of pulmonary nocardiosis," The Indian Journal of Chest Diseases \& Allied Sciences, vol. 41, no. 3, pp. 153 157, 1999.

[25] R. Kumar, D. Chhina, V. Kaushal, R. Mahajan, and H. Kaur, "Cytological diagnosis of pulmonary nocardiosis in an immunocompromised patient," Indian Journal of Medical Microbiology, vol. 26, no. 4, pp. 380-382, 2008.

[26] P. F. Long, "A retrospective study of Nocardia infections associated with the acquired immune deficiency syndrome (AIDS)," Infection, vol. 22, no. 5, pp. 362-364, 1994.

[27] V. Wadhwa, S. Rai, P. Kharbanda, S. Kabra, R. Gur, and V. K. Sharma, "A fatal pulmonary infection by Nocardia brasiliensis," Indian Journal of Medical Microbiology, vol. 24, no. 1, pp. 63-64, 2006.

[28] R. J. Wallace Jr., "Treatment options for nocardial infection in HIV patients," Journal of critical illness, vol. 8, article 768, 1993.

[29] S. J. Burgert, "Nocardiosis: a clinical review," Infectious Diseases in Clinical Practice, vol. 8, no. 1, pp. 27-32, 1999.

[30] K. Gowrinath, W. W. Baig, A. R. Prabhu, K. Chawla, and I. Bairy, "Pulmonary nocardiosis due to Nocardia farcinica in a renal transplant recipient," The Indian Journal of Chest Diseases \& Allied Sciences, vol. 51, no. 4, pp. 237-239, 2009. 


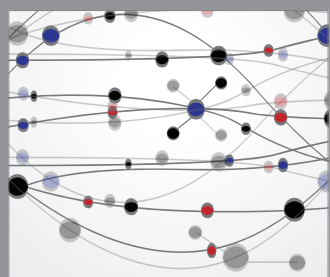

The Scientific World Journal
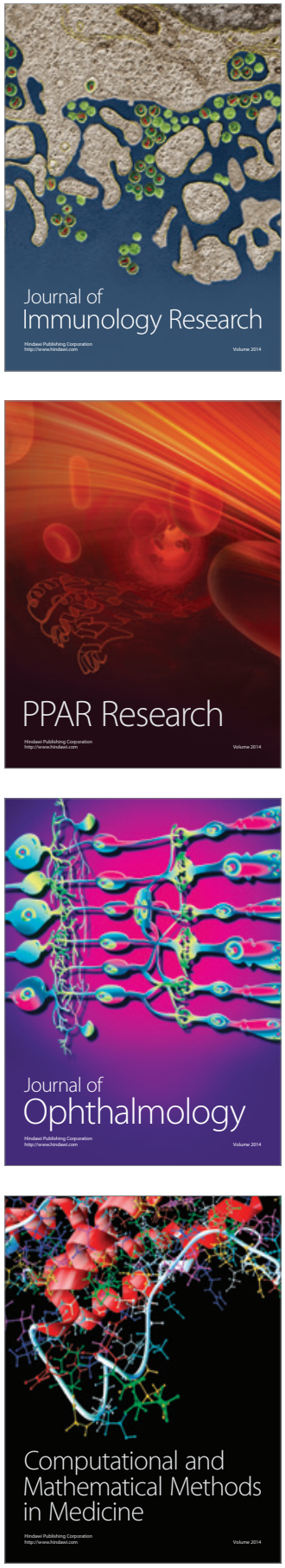

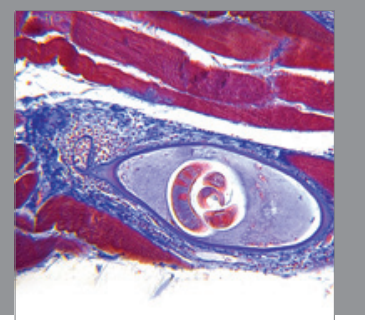

Gastroenterology

Research and Practice
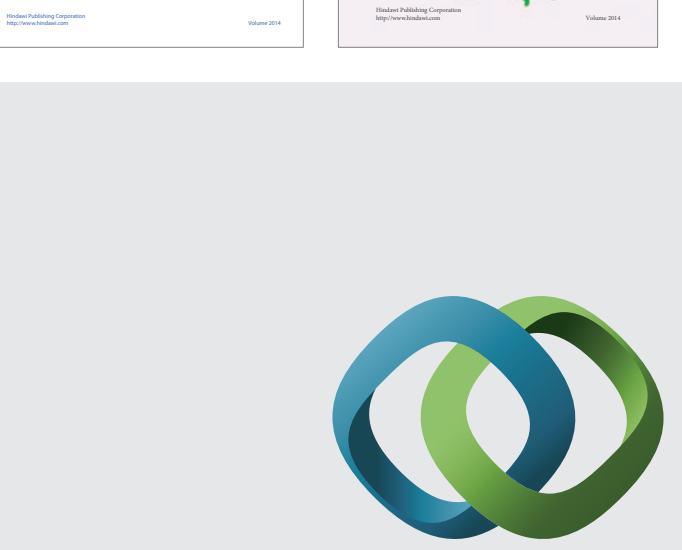

\section{Hindawi}

Submit your manuscripts at

http://www.hindawi.com
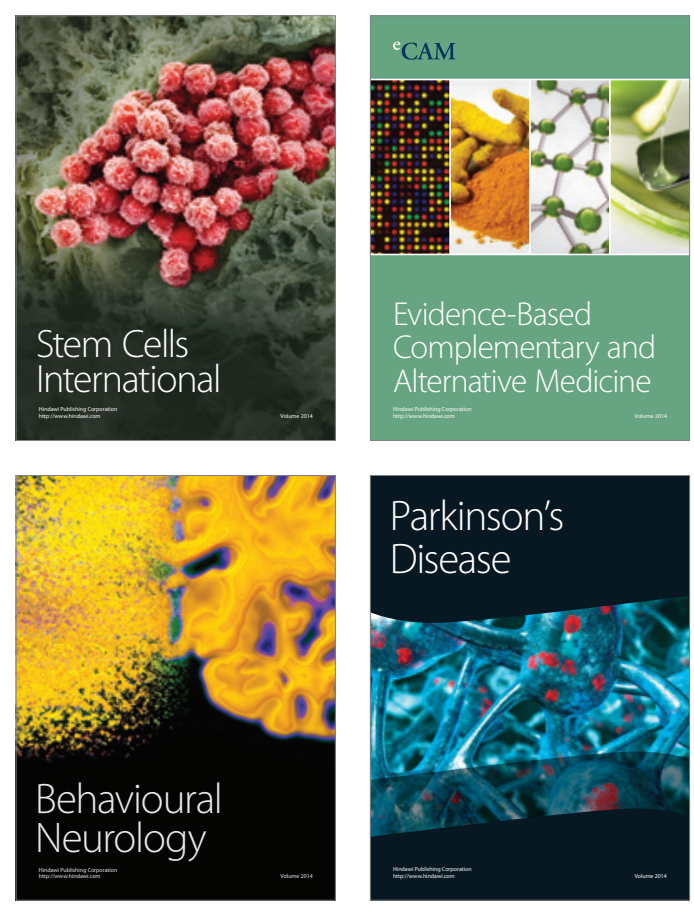

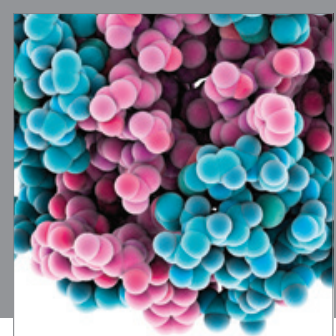

Journal of
Diabetes Research

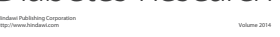

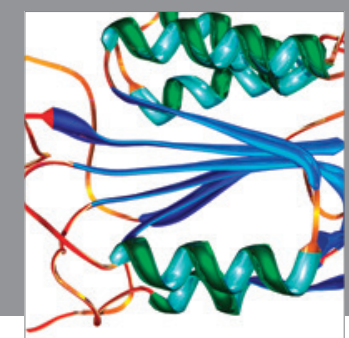

Disease Markers
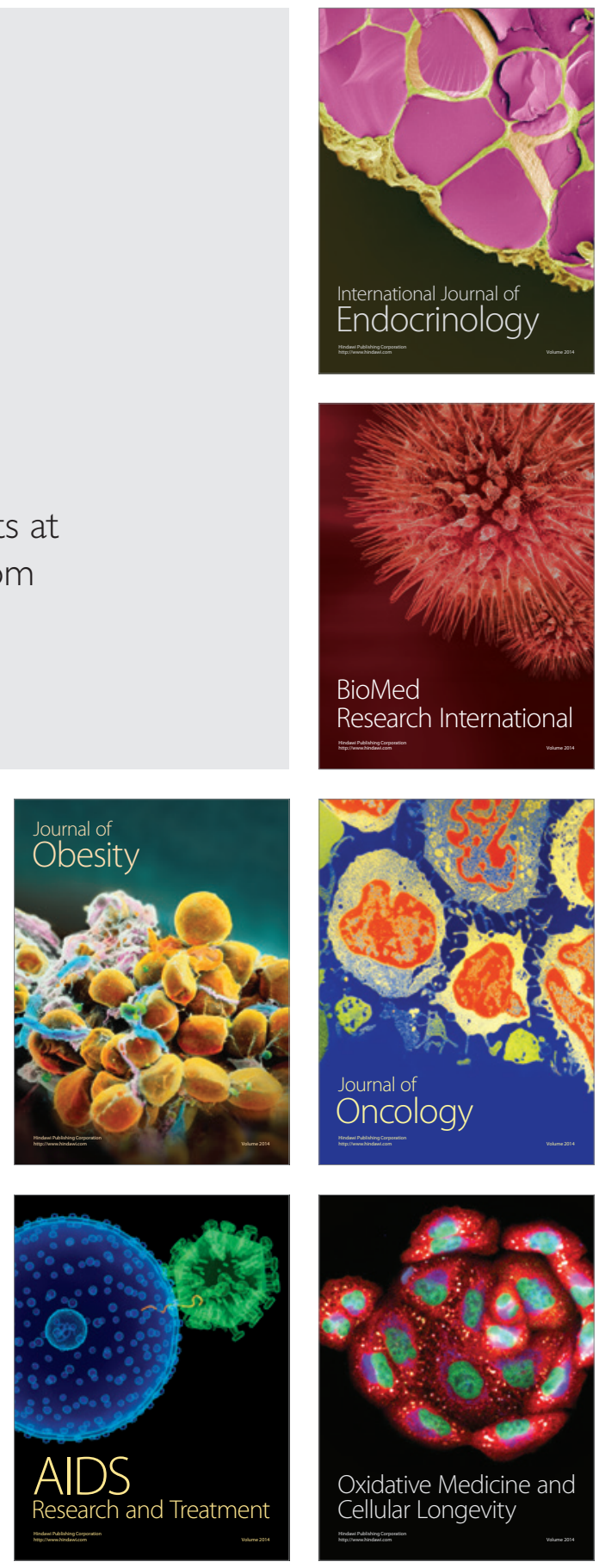